\title{
Improved Stochastic Random Walker Segmentation based on Gaussian Filtering
}

\author{
Yogendra Kumar Jain \\ Head of Department \\ Computer Science \& Engineering Department \\ Samrat Ashok Technological Institute Vidisha
}

(M.P)

\author{
Nitin Kumar Patel \\ Research Scholar \\ Computer Science \& Engineering Department \\ Samrat Ashok Technological Institute Vidisha
}

(M.P)

\begin{abstract}
Image segmentation is the process to capture the object from the background and it is a difficult task when a vision of the object is in stochastic region. Here introduce in this paper extension of stochastic random walker segmentation method. In stochastic random walker segmentation, a weighted graph is built from the image, where the each pixel considered as a node and edge weights depend on the image gradient between the pixels. For given seed regions, the probability are evaluated for a stochastic random walk on this graph starting at a pixel to end in one of the seed regions. The problem associated with existing method is the number of random variable (gray-level value in random order) in stochastic images. These random variables increase the graph sizes of stochastic images. If the graph size will increase, consequently execution time would also increase. To overcome these problems, the proposed "Improved stochastic random walker segmentation based on Gaussian filtering" for stochastic image segmentation. In proposed method Gaussian filter has been used for the removal of uncertain gray level and which in turn reduce the noise level and the resultant graph size of corresponding stochastic image, then apply stochastic random walker segmentation method which may help to decrease the execution time of the segmentation process.
\end{abstract}

\section{Keywords}

Gaussian filter, Interactive image segmentation, Stochastic Random walker segmentation, canny edge detection.

\section{INTRODUCTION}

Image segmentation is the initial step of image analysis, the principle of image segmentation is to partition an image into meaningful regions with respect to a particular application. Image segmentation typically on noisy image as shown in (figure 1) is a critical work. Image noise depends on the image acquisition process e. g. Digital camera, magnetic resonance, computed tomography and ultrasound device etc. in the process of image acquiring must be informed about the measurement error. These errors responsible for loss of information and influences the result of the image segmentation. The discussion of error propagation is important in, for example, in a field of medical purpose, where decisions about patient treatments are based on quantitative information extracted from medical images. Treatment decisions for chemotherapy patients are based on the improvement of the volume of the segmented lesions from the noisy images. Thus, it is important to measure the extracted data with a reliable estimate.
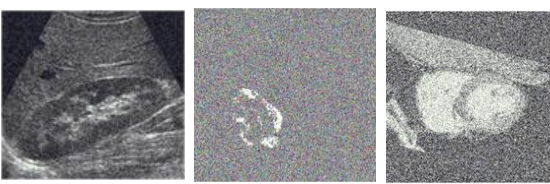

Figure 1. Noisy images contain uncertain gray-level

Many developments are offered in the field of image segmentation techniques without human interaction, but still are not getting a much an efficient scheme. Due to large invention of such techniques are still going on, it is always required to semi supervised method for solving real time problem domain. Interactive segmentation algorithms are suitable for any application domain. However, applications on mobile touch screen devices put extra constraints on the algorithm. Due to the imprecision of user inputs on small screens, such algorithms should be able to tolerate some errors. As well it is also attractive not to include any correction stage of the algorithm in order to avoid tedious zooming and correction processes [1]

Random walker algorithm [2] is one the approach of interactive segmentation method, there is no information about included uncertainties, however its name might suggest random walker is chosen because the direction of the random walk is randomly chosen from probabilities based on the deterministic image gradient. Considerably, error propagation is hard or even unfeasible for most of the image processing algorithms including the random walker method. Now this method replaces with stochastic random walker segmentation that solve problem of error propagation.

The stochastic random walker segmentation method [3] consider the pixel values to be random variables (RVs), thus introducing the concept of stochastic images. Then wind up with stochastic weights for the graph in random walker segmentation and a stochastic partial differential equation (PDE) has to be solved. Then discretize the RVs and the stochastic PDE by the method of generalized polynomial chaos, relating the recent developments in numerical methods for the discretization of stochastic partial differential equation and an interactive segmentation algorithm. The resulting algorithm involve for the detection of regions where the segmentation result is highly affected by the uncertain pixel values. Thus, it gives a reliability estimate for the resulting segmentation, and it additionally allows determining the probability density function of the segmented object volume.

The remaining part of the paper is organized as follows: In section 2 give reviews of literature of related work. Section 3 covers the overview of the stochastic random walker 
segmentation algorithm. Section 4 describes the proposed methodology. In section 5, the result analysis has been carried out. Section 6 provides performance evaluation of the proposed work with the existing technique. Finally section 7 concludes the paper.

\section{REVIEW OF LITERATURE \\ 2.1 Interactive Segmentation}

Interactive image segmentation is a semi-automatic image segmentation technique. This type of segmentation technique allows minimal user interaction in the segmentation process. For a good interactive image segmentation algorithm, there are two basic needs: first is given a certain user input, and the algorithm should produce perceptive segmentation that reflects the user objective, and the second is the algorithm must be efficient so that it can provide instant visual response. Interactive segmentation methods in the literature can be divided into two main classes, as boundary-based [4] and region-based methods [5-8]. Boundary based methods require the user to select an approximate boundary around the object, and then try to find the correct boundary. Region based segmentation algorithms operate iteratively, gathering pixels that are neighbors and have similar values and splitting groups of pixels are dissimilar in value.

Graph cut based methods, an image is modeled as graph where each node corresponds to a pixel and two neighboring nodes are connected with a weighted edge defined as the distance between the pixel values. Furthermore, the graph cut algorithm [9], models object and background pixel values according to histograms. Based on graph cut there are other methods such as Dynamic and Iterated Graph-Cut and Image Segmentation Using Minimal Graph Cuts [10]. The drawback of graph cut based algorithm is that it does not hold fine shapes and this method does not well when the level of noise is more.

The random walker algorithm achieves better performance as compared to segmentation by graph cut algorithm. Similarly, the random walker algorithm requires the input of object and background seeds. However, the random walks algorithm is essentially an approach that minimizes energy dirichlet boundary conditions, where different boundary conditions (different entrance seeds) always lead to different harmonic functions. This method is inefficient when apply on noisy or stochastic images. The random walker segmentation method is extending with stochastic images.

\subsection{Gaussian Filter}

The Gaussian filter is very effective tool for removing noise and preserving the property of the image. Gaussian smoothing is a 2-D convolution operator. The degree of smoothing is resolved by the standard deviation of the Gaussian (larger standard deviation Gaussian obviously requires larger convolution kernels in order to be accurately represented.). Gaussian filter is also used for nonlinear filtering problem such as stochastic process [11].

While working with images, we require to use the two dimensional Gaussian function. This is simply the product of two 1D Gaussian function and is given by

$$
G(A, B)=\frac{1}{2 n \sigma^{2}} e^{-\frac{A^{2}+B^{2}}{2 \sigma^{2}}}
$$

Where $\sigma$ represents the standard deviation of the distribution, $\mathrm{A}$ and $\mathrm{B}$ is the dimension of an image. $\mathrm{A}$ is the distance from the origin in the horizontal axis; $\mathrm{B}$ is the distance from the origin in the vertical axis.

\section{OVERVIEW OF STOCHASTIC RANDOM WALKER SEGMENTATION}

The addition of the random walker segmentation to stochastic images is straightforward and follows the methods for processing of stochastic images described by Preusser et al. [12] and [13]. In stochastic random walker segmentation, classical images are replaced by stochastic images.

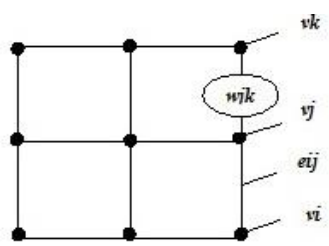

Figure 2. Graph generated from an image contains 9 nodes and 12 edges. Every edge $e i j$ has a weight $w i j$

The edge weights for the graph are defined by the difference of two RVs (pixel), which describe the grayvalue uncertainty for the corresponding pixels. Then end up with stochastic edge weights, which are random variable (RVs), i.e. the discretization of stochastic images use the generalized polynomial chaos (GPC) extension developed in [14].

The GPC extension provides a basis for the space of square integrable RVs, built by polynomials in RVs with known distribution. This enables us to deal with deterministic coefficients to represent RVs in the GPC, because the basis functions encode the stochasticity and produce a stochastic partial differential equation (SPDEs). The resulting SPDEs are solved using the generalized spectral decomposition (GSD) [15], yielding to a tractable and fast algorithm for the high-dimensional stochastic problems.

\section{PROPOSED METHOD}

Stochastic random walker segmentation has the problem of a number of random variable (pixel gray-level in random order of stochastic image), stochastic images which contain additive noise, these noise increase uncertainty level of the images. It causes the increase execution time of the segmentation process. Now there is a need of an efficient technique to resolve problem of random variable. To overcome these problems, proposed a method to detect object from stochastic images using the proposed improved stochastic random walker segmentation based on Gaussian filtering. The proposed method gives better result in terms of sharpness, computation time and optimal tracking boundary of the object. The block diagram of proposed algorithm is representing in figure 3 .

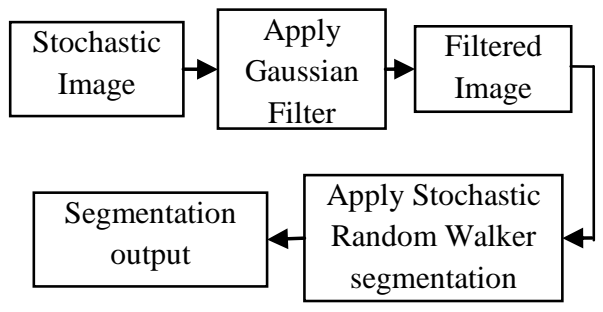


Figure 3. Block diagram of proposed algorithm

Steps of the proposed algorithm are as follows:

step1: Read stochastic images which are to be segmented. step2: Use Gaussian filter for reducing the number of Random variables of stochastic images.

step3: Then treat pixels of image as a random variables, the discretization of RVs utilizes generalized polynomial chaos (GPC).

step4: GPC enable us to deal with deterministic coefficient because the basic functions encode the stochasticity and produce stochastic partial differential equation (SPDE). step5: These SPDE are solved using generalized spectral decomposition combining with interactive segmentation techniques. step6: Detection of object region from segmentation method.

step7: For detection of optimal object boundary, used canny edge detection.

Improved stochastic random walker segmentation provides better results in terms of optimal detection of objects and its boundary. The proposed method also has the ability to detect objects when the level of noise more in stochastic images

\section{RESULT ANLYSIS}

Here illustrate the benefits of the improved stochastic random walker segmentation based on Gaussian filtering. This approach reduces the noise level of the input image. To start with it, first of all get an axial image that we have to be segment. This image contains a number of uncertain gray-level and noise. Then apply a Gaussian filter of size $[5,5]$ with standard deviation $(\sigma=2)$, it would reduce the unwanted pixel of an image and produced resultant image after reduction of noise. Stochastic random walker segmentation is region based interactive segmentation, so we need to defined seed points and expected region for starting segmentation process.

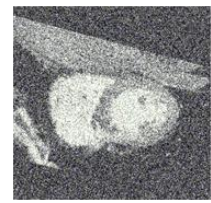

(a)

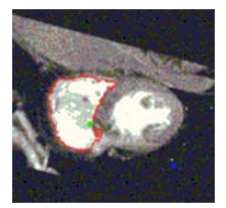

(c)

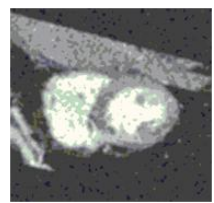

(b)

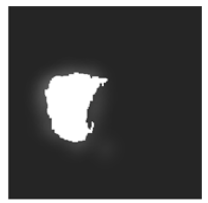

(d)
Figure 4. (a) Axial image (b) Resultant image after filtering(c) User defined seed point green for object and blue for the background (d) Segmentation output.

Now define two seed points likewise for object and background. As shown in figure 4(c) two user defined seed points, green for object part and blue for background part. Then apply segmentation method to end with obtaining segmented region in figure 4(d) of image.

In figure 5 demonstrate the proposed result on the ultrasound kidney image. A similar procedure will apply on ultrasound image as previously applied to axial image. The choice of the parameter $\sigma$ depends on the stochastic images, if the image contains more noise than increase value of $\sigma$ (smoothing factor). Figure 5(a) shows an ultrasound kidney image which contains noise and gray level in random order.

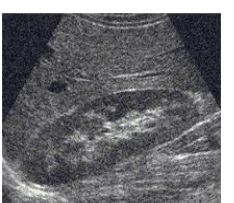

(a)

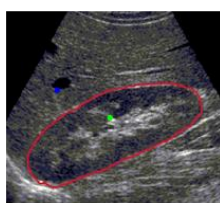

(c)

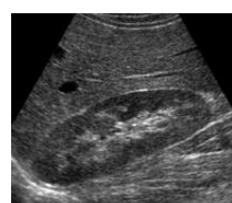

(b)

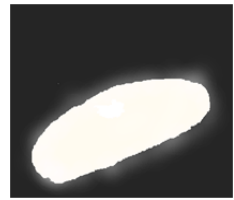

(d)
Figure 5. (a) Ultrasound image. (b) Resulting image after Gaussian filtering

(c) The user defines seed point green for object and blue for background. (d) Segmentation output.

In this image now apply a Gaussian filter of size $[5,5]$ and value of smoothing factor $\sigma=1$ (in case of ultrasound image) and obtained figure 5(b) which is the resultant image after filtering. Then define seed points one for object level and other for background level as previously mention like axial image. In figure 5(c) apply stochastic random walker segmentation method that described in section 3 and the segmentation result of ultrasound image is obtained in figure 5(d).

Here compare the result of improved stochastic random walker segmentation based on Gaussian filtering with the original stochastic random walker segmentation. The comparison is based on the expected execution time and sharpness of the detected region of segmentation of both filtered image and stochastic image. The proposed method gives better performance in terms of execution time and sharpness of object boundary detected.

Now show result comparison between proposed and existing method based on the object detection in liver image. Liver image as shown in figure 6 (a) is directly segmented through stochastic random walker segmentation method and result of segmentation is obtained in figure 6 (b).

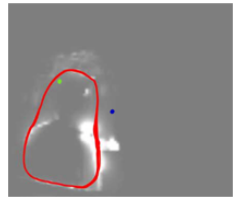

(a)

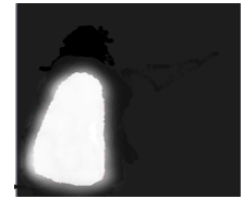

(b)
Figure 6. Segmentation result based on Existing method (a) Liver image (b) Segmentation output

The segmentation output of existing method has uncertainty in terms of object boundary. Now apply [5,5] Gaussian filter with $\sigma=1$ on same liver images for reducing the noise level, and produce filtered image as shown in figure 7 (a). Then apply segmentation method and final result of segmentation is obtained in figure 7(b). 


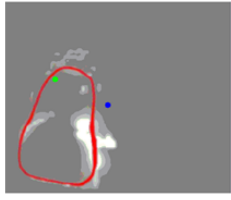

(a)

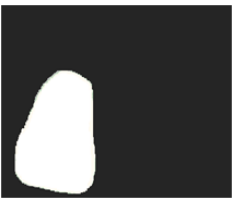

(b)
Figure 7 Segmentation result based on proposed method

(a) Filtered liver image (b) segmentation output

The proposed method needs to detect the deterministic object location. To capture the exact volume of object and optimally tracking object boundary, now use canny edge detection [16]. The aim of boundary detection is generally substantially reduces the amount of data that can be used in segmented part while maintaining the structural properties for subsequent part of the object. As shown in figure 8 stochastic object boundary can be visualized into deterministic object boundary.
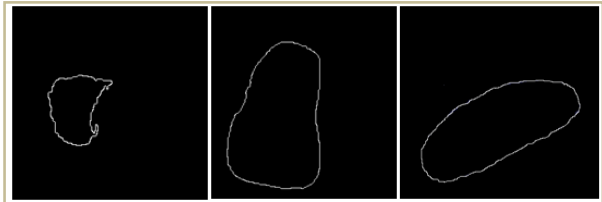

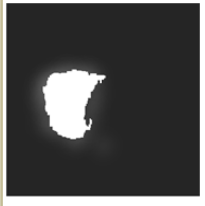

Axial

Image

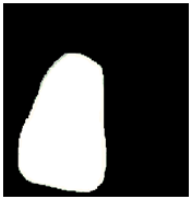

Liver

Image

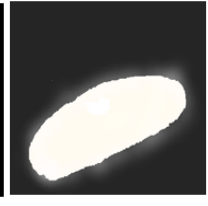

Ultrasound Image
Figure 8. The stochastic object boundary can be visualized by tracking the deterministic object boundary through canny edge detection.

\section{PERFORMANCE EVAlUATION}

It is always difficult to capture object location form stochastic environment. Performance evaluation of the segmentation method can be analyzed through some parameters. These parameters are computation time of the segmentation process, peak signal to noise ratio and mean square error of region detection through segmentation process. These parameters can contribute to show how proposed method is better than existing method.

\subsection{Execution Time}

The comparison of proposed results on the basis of expected execution time of the process of segmentation, of all three images. Here compare the execution time between existing and the proposed method. The benefit of proposed techniques is significantly reduces the execution time of the segmentation process.

Table 1 represents the comparison of execution time between the proposed method and existing method. In existing method, execution time exponentially grows with their RVs in stochastic images. But in the proposed technique, observe that execution time is decreases, because utilizing the Gaussian filtering approach before applying the segmentation method. Reduction of the execution time is proportional to reduction of number of $\mathrm{RVs}$ in stochastic images. It also helps for enhancing the visibility of detected region.
Table 1. Comparison of execution time (in second) of the segmentation method applied on Axial, Ultrasound, Liver images

\begin{tabular}{|c|c|c|}
\hline Images & $\begin{array}{c}\text { Existing } \\
\text { Method }\end{array}$ & $\begin{array}{c}\text { Proposed } \\
\text { Method }\end{array}$ \\
\hline $\begin{array}{c}\text { Axial } \\
\text { Image }\end{array}$ & 3.52 & 2.66 \\
\hline $\begin{array}{c}\text { Ultrasound } \\
\text { Image }\end{array}$ & 6.53 & 4.83 \\
\hline $\begin{array}{c}\text { Liver } \\
\text { Image }\end{array}$ & 5.56 & 4.22 \\
\hline
\end{tabular}

\subsection{PSNR and MSE}

Result of the proposed method analyzes with the help of two errors metric PSNR \& MSE. PSNR measure a peak error and MSE represents the cumulative squared error. Now illustrate a comparison of MSE \& PSNR ratio between results of segmentation by stochastic random walker segmentation with the improved stochastic random walker segmentation based on Gaussian filtering.

The comparison of the MSE and PSNR values between the proposed and existing method are shown in Table 2 and 3. The proposed method has high PSNR value and low MSE value as compare to exiting method. It clearly depicted that object detected through the proposed method is enhanced. The proposed method is useful particularly where gray value uncertainty is more in stochastic images.

Table 2. Comparison of the PSNR values (in decibel) on the detected segmented region of images

\begin{tabular}{|c|c|c|}
\hline Images & $\begin{array}{c}\text { Existing } \\
\text { Method }\end{array}$ & $\begin{array}{c}\text { Proposed } \\
\text { Method }\end{array}$ \\
\hline $\begin{array}{c}\text { Axial } \\
\text { Image }\end{array}$ & 30.21 & 33.08 \\
\hline $\begin{array}{c}\text { Ultrasound } \\
\text { Image }\end{array}$ & 30.38 & 32.92 \\
\hline $\begin{array}{c}\text { Liver } \\
\text { Image }\end{array}$ & 30.46 & 33.03 \\
\hline
\end{tabular}

Table 3. Comparison of the MSE value of the detected segmented region of images

\begin{tabular}{|c|c|c|}
\hline Images & $\begin{array}{c}\text { Existing } \\
\text { Method }\end{array}$ & $\begin{array}{c}\text { Proposed } \\
\text { Method }\end{array}$ \\
\hline $\begin{array}{c}\text { Axial } \\
\text { Image }\end{array}$ & 62.50 & 32.37 \\
\hline $\begin{array}{c}\text { Ultrasound } \\
\text { Image }\end{array}$ & 59.98 & 33.21 \\
\hline $\begin{array}{c}\text { Liver } \\
\text { Image }\end{array}$ & 59.26 & 32.55 \\
\hline
\end{tabular}

\section{CONCLUSION}

The proposed technique provides efficiencies in terms of computational time as well as the sharpness of the segmented part of the image. The existing technique implemented for the segmentation of stochastic images using stochastic random walker segmentation provides less detection of the segmented part since the level of noise is more. Hence in the proposed work by applying filtering technique, noisy level can be reduced and for the sharpness of the segmented region, canny edge detector is applied. Apart from all these application, there is open question for future research is the starting point of the segmentation process is similar to classical random walker segmentation. 


\section{REFERENCES}

[1] Sener Ozan, Ugur Kemal an Aydin Alatan A. 2012. Error-tolerant Interactive Image Segmentation using Dynamic and Iterated Graph-Cuts. Proceedings of the 2nd ACM international workshop on Interactive multimedia on mobile and portable devices, pp.9- 16 .

[2] Grady L. 2006. Random walks for image segmentation. IEEE Transaction. Pattern Anal. Mach. Intell. Vol14-No11, pp.1768-1783.

[3] Pätz Torben and Preusser Tobias. Segmentation of Stochastic Images with a Stochastic Random Walker Method. 2012. IEEE Transactions on Image Processing. Vol21-No5, pp.2424-2433.

[4] Li Y., Sun J., Tang C. -K., and Shum H. -Y. 2004. Lazy snapping. ACM Transactions on Graphics (TOG). vol.23 issue.3, pp.303-308.

[5] Calderero F. and Marques F. 2010. Region merging techniques using information theory statistical measures. IEEE Transactions on Image Processing. vol 19. issue.6, pp.1567-86.

[6] Liu D., Pulli K., Shapiro L. G., and Xiong Y. 2010. Fast interactive image segmentation by discriminative clustering. Proceedings in ACM multimedia workshop on Mobile cloud media computing. pp.4752.

[7] Liu D., Xiong Y., Shapiro L.G., and Pulli K. 2009. Robust interactive image segmentation with automatic boundary refinement. in ICIP, pp. 225-228.

[8] Xue Ya, Liao Xuejun and Carin Lawrence. 2007. Multi-Task Learning for Classification with Dirichlet Process Priors, Journal of Machine Learning Research vol. 8 , pp. $35-63$.
[9] Boykov Y. and Jolly M.-P. 2001. Interactive graph cuts for optimal boundary and region segmentation of objects in N-D images. In ICCV, pp.105-112.

[10] Eriksson Anders P., Barr Olof and Astrom Kalle .Image Segmentation Using Minimal Graph Cuts. Proceedings in CiteSeerX International Journal of Image Processing (IJIP).

[11] Ito K. 2000. Gaussian filters for nonlinear filtering problems Automatic Control. IEEE Transactions on vol.45, issue. 5 pp. $910-927$.

[12] Preusser T., Scharr H., Krajsek K. and Kirby R.M. 2008. Building blocks for computer vision with stochastic partial differential equations. Int J. Comput. Vis., Vol80-No3, pp. 375-405.

[13] Pätz T. and Preusser T. 2010. Ambrosio-Tortorelli segmentation of stochastic images. Proceeding in ECCV. vol.6315, pp.254-267.

[14] Debusschere B.J., Najm H.N., Pébay P.P., Knio O.M., Ghanem R. G., and Le Martre O. P. 2005. Numerical challenges in the use of polynomial chaos representations for stochastic processes. SIAM J. Sci. Comput., Vol26-No.2, pp. 698-719.

[15] Nouy A. 2007. A generalized spectral decomposition technique to solve a class of linear stochastic partia differential equations. Comput. Methods Appl. Mech. Eng., Vol196-No.45-48, pp.4521-4537.

[16] Canny John. 1986. A computational approach to edge detection. Pattern Analysis and Machine Intelligence. IEEE Transactions on PAMI-8, pp.679-698. 